\title{
Research Exploring Physical Activity in Care Homes (REACH): study protocol for a randomised controlled trial
}

\author{
Anne Forster ${ }^{1 *}$, Jennifer Airlie ${ }^{1,2}$, Karen Birch², Robert Cicero ${ }^{3}$, Bonnie Cundill $^{3}$, Alison Ellwood ${ }^{4}$, Mary Godfrey ${ }^{1}$, \\ Liz Graham ${ }^{3}$, John Green ${ }^{4}$, Claire Hulme ${ }^{5}$, Rebecca Lawton ${ }^{6}$, Vicki McLellan ${ }^{3}$, Nicola McMaster ${ }^{4}$, Amanda Farrin $^{3}$, \\ on behalf of the REACH Programme Team
}

\begin{abstract}
Background: As life expectancy increases and the number of older people, particularly those aged 85 years and over, expands there is an increase in demand for long-term care. A large proportion of people in a care home setting spend most of their time sedentary, and this is one of the leading preventable causes of death. Encouraging residents to engage in more physical activity could deliver benefits in terms of physical and psychological health, and quality of life. This study is the final stage of a programme of research to develop and preliminarily test an evidence-based intervention designed to enhance opportunities for movement amongst care home residents, thereby increasing levels of physical activity.

Methods/design: This is a cluster randomised feasibility trial, aiming to recruit at least 8-12 residents at each of 12 residential care homes across Yorkshire, UK. Care homes will be randomly allocated on a 1:1 basis to receive either the intervention alongside usual care, or to continue to provide usual care alone. Assessment will be undertaken with participating residents at baseline (prior to care home randomisation) and at 3, 6, and 9 months post-randomisation. Data relating to changes in physical activity, physical function, level of cognitive impairment, mood, perceived health and wellbeing, and quality of life will be collected. Data at the level of the home will also be collected and will include staff experience of care, and changes in the numbers and types of adverse events residents experience (for example, hospital admissions, falls). Details of National Health Service (NHS) usage will be collected to inform the economic analysis. An embedded process evaluation will obtain information to test out the theory of change underpinning the intervention and its acceptability to staff and residents.
\end{abstract}

Discussion: This feasibility trial with embedded process evaluation and collection of health economic data will allow us to undertake detailed feasibility work to inform a future large-scale trial. It will provide valuable information to inform research procedures in this important but challenging area.

Trial registration: ISRCTN registry, ISRCTN16076575. Registered on 25 June 2015.

Keywords: Care homes, Elderly, Feasibility, Cluster randomised controlled trial, Physical activity, Movement, Sedentary

\footnotetext{
* Correspondence: A.Forster@leeds.ac.uk

${ }^{1}$ Academic Unit of Elderly Care and Rehabilitation, Bradford Teaching

Hospitals NHS Foundation Trust and University of Leeds, Bradford, UK

Full list of author information is available at the end of the article
} 


\section{Background}

Life expectancy has increased dramatically over the last century. While policy and service developments have emphasised alternatives to long-term care, evidence suggests that around one in four older people will spend time in a care home in their last year of life [1] and that the need for such care will persist [2]. In 2011, more than a quarter of a million $(291,000)$ people aged 65 years and over were living in care homes in England and Wales, 3.2\% of this population [3].

Research over decades reports that care home residents spend the majority of their time inactive [4]. Lack of engagement in physical activity (PA), defined as "any bodily movement produced by skeletal muscles that results in energy expenditure" [5], has detrimental effects on physical and psychological health and quality of life, and contributes to social isolation [6]. Additionally, an observational study suggests that $97 \%$ of residents' days are spent sedentary (e.g. sitting, watching television), with low levels of interaction with staff and each other [4]. Sedentary behaviour, defined as "any waking behaviour characterised by an energy expenditure $\leq 1.5$ metabolic equivalents (METs) while in a sitting or reclining posture" [7], has been seen to negatively impact morbidity and mortality independently of PA [8]. Consequently, the National Institute for Health and Care Excellence (NICE) [9] issued a quality standard call for "older people in care homes to be offered opportunities during their day to participate in meaningful activity that promotes their health and mental wellbeing". Meaningful activity can range from activities such as caring for plants and helping around the home, to leisure pursuits such as playing cards.

Existing research has primarily involved the delivery of time-limited interventions by staff external to the care homes [10]. It seems necessary that, if such interventions are to be successfully and sustainably delivered, they need to be embedded in routine practice and that care home staff should be involved in developing and delivering the necessary change [11]. Further, there is increasing emphasis on programmes which reduce the overall time spent sedentary [12] and that do not simply involve short bursts of formally organised PA such as exercise classes. Together, these reinforce the need for action to increase levels of PA in care homes, reduce time spent sedentary, and incorporate greater engagement of care home staff in developing and delivering whole practice change which is embedded in daily life routines.

Through a series of studies we have collaborated with care home staff and residents to develop a multicomponent intervention aimed at changing how the routine work of care staff is carried out to encourage and support residents to engage in more PA (above and beyond organised exercise sessions) and reduce the time they spend sedentary. Increasing resident PA and reducing the time they spend sedentary will, we hypothesise, improve residents' physical and psychological outcomes.

The first study for the Research Exploring Physical Activity in Care Homes (REACH) programme involved ethnographic fieldwork in four care homes to understand the contextual and organisational factors that shape care practice, residents' existing patterns of PA, and the potential for change. It emerged in this first study, and was reinforced in later studies, that 'physical activity' was a difficult concept for care home staff to understand and engage with, particularly in view of the frailty of many residents. Staff linked the notion of PA with specific time-limited activities which might be undertaken by an activity co-ordinator. Our intent was to engage all staff in encouraging residents to undertake small changes in their daily life. The notion of increasing movement and 'moving more' had greater resonance and intuitive understanding for staff and residents. Thus, this is the language we adopted in the care home setting. We anticipate that 'moving more' will increase levels of PA.

The second study explored the acceptability, validity, and reliability of different measures of PA and sedentary behaviour, which included the development and testing of protocols for the use of accelerometers by residents.

In the third study, the researchers worked alongside a reference group of care home managers, staff, residents, and their relatives/friends to identify barriers and opportunities for change through the process of intervention mapping [13].

In the fourth study, through an action research approach with care home staff and residents in a further four homes, we developed and tested elements of an intervention and change process to reduce residents' sedentary time and encourage them to 'move more'. An iterative approach, drawing on the previous work and the implementation literature, was utilised during this process. A novel feature of the work was the involvement of an artist to capture and illustrate pictorially the voice of residents, which resonated with staff in a way that words did not.

Here, we report the protocol for the fifth study, designed to explore the feasibility of training staff to incorporate the intervention into their care home, and the feasibility of trial processes (recruitment, follow-up, data collection) to inform the feasibility and design of a future definitive randomised controlled trial (RCT).

\section{Methods/design \\ Design}

The REACH feasibility trial is a pragmatic, multicentre, cluster-randomised controlled trial to explore the practicality and acceptability of implementing a large-scale definitive cluster $\mathrm{RCT}$ comparing the $\mathrm{REACH}$ intervention 
plus usual care versus usual care practice alone among permanent residents living in residential care homes in the UK. The REACH intervention is a whole-home intervention designed to assist care home staff to make step-by-step changes in their approach to working with residents, and therefore the care home is the unit of randomisation.

Figure 1 illustrates the timing of all trial processes (Additional file 1).

\section{Objectives}

The study objectives relate to the feasibility trial and the linked process evaluation. The former will involve gathering data around recruitment and follow-up, intervention delivery, assessment of outcome measures, and statistical aspects to inform the feasibility and design of a definitive RCT. The process evaluation, drawing on Medical Research Council guidance on process evaluations [14], will collect data relating to the 'how' of the intervention and implementation process (the 'theory of change' or assumptions underpinning the programme); the process and quality of implementation as it evolves in real time [15]; and how implementation impacts on, and is shaped by, the organisational and interactional environment of the care home [16].

In summary, the main objectives are to:
1. Explore the optimum strategies to facilitate recruitment, and estimate recruitment and follow-up rates at both care home and resident levels;

2. Assess the compliance and feasibility of wearing an accelerometer to obtain data to measure the level of a resident's PA (anticipated primary outcome for the definitive RCT) and time spent sedentary;

3. Evaluate the most appropriate outcome measures by assessing the feasibility of obtaining questionnaire data among residents related to physical function, ability and mobility, and psychological well-being;

4. Determine the optimum strategy for collecting data relating to health care resource use and adverse events. The reliability of routinely collected data at both the care home and resident level will also be considered;

5. Assess intervention fidelity in terms of exposure, reach, and quality of implementation, as well as the acceptability to staff and their understanding of the intervention;

6. Explore factors contributing to and hindering organisational and practice change, and the contextual factors that affect variation in the outcomes achieved;

7. Investigate the characteristics of usual care within residential care homes;

\begin{tabular}{|c|c|c|c|c|c|c|}
\hline \multirow[b]{3}{*}{ TIMEPOINT } & \multicolumn{6}{|c|}{ STUDY PERIOD } \\
\hline & \multirow{2}{*}{$\begin{array}{c}\begin{array}{c}\text { Screening \& } \\
\text { resident } \\
\text { recruitment }\end{array} \\
-2-0 \\
\end{array}$} & \multirow{2}{*}{$\begin{array}{c}\begin{array}{c}\text { Care Home } \\
\text { randomisation }\end{array} \\
0 \\
\end{array}$} & \multicolumn{3}{|c|}{$\begin{array}{c}\text { Post-randomisation } \\
\text { (months) }\end{array}$} & \multirow{2}{*}{$\begin{array}{c}\text { Close-out } \\
10\end{array}$} \\
\hline & & & 3 & 6 & 9 & \\
\hline \multirow{4}{*}{$\begin{array}{r}\text { ENROLMENT: } \\
\text { Care Home eligibility } \\
\text { Resident eligibility } \\
\text { screening } \\
\text { Capacity assessment }\end{array}$} & & & & & & \\
\hline & $x$ & & & & & \\
\hline & $x$ & & & & & \\
\hline & $x$ & & & & & \\
\hline \multirow{3}{*}{$\begin{array}{r}\text { Consent / assent } \\
\text { Baseline data } \\
\text { collection } \\
\text { Allocation }\end{array}$} & $x$ & & & & & \\
\hline & $x$ & & & & & \\
\hline & & $x$ & & & & \\
\hline \multirow{3}{*}{$\begin{array}{r}\text { INTERVENTIONS: } \\
\text { Usual care } \\
\text { REACH intervention } \\
\text { (Move More) }\end{array}$} & & & & & & \\
\hline & & & $\leftarrow$ & & $\rightarrow$ & \\
\hline & & & $\leftarrow$ & & $\rightarrow$ & \\
\hline \multicolumn{7}{|l|}{ ASSESSMENTS*: } \\
\hline $\begin{array}{r}\text { Resident screening data } \\
\text { (anonymized) }\end{array}$ & $x$ & & & & & \\
\hline Home level data & & & $x$ & $x$ & $x$ & $x$ \\
\hline Staff level data & & & $x$ & $x$ & $x$ & $x$ \\
\hline $\begin{array}{r}\text { Resident level data } \\
\text { (researcher interview or proxy) }\end{array}$ & & & $x$ & $x$ & $x$ & $x$ \\
\hline
\end{tabular}

Fig. 1 SPIRIT figure 
8. Provide a preliminary estimate of the effectiveness of the intervention as measured by the volume and level of PA undertaken and the time spent sedentary from the accelerometer data;

9. Calculate reliable estimates to inform the sample size calculations for a definitive trial by assessing the variability in study outcomes in both arms, estimating the intra-cluster correlation coefficient (ICC), and estimating likely cluster size and corresponding between-cluster variability;

10. Investigate the within-trial and long-term incremental cost effectiveness of the intervention compared with usual care.

\section{Recruitment setting and participants}

The study will be conducted in 12 residential care homes in the counties of West and North Yorkshire in the UK. A care home will be considered eligible if:

1) Initial scoping indicates that there are sufficient numbers of permanent eligible residents to enable 8-12 permanent residents to be recruited from that home (homes will later be excluded if researchers find that they are unable to obtain consent and baseline data for at least 5 residents); and

2) There is a manager or nominated person who agrees to sign up to the trial protocol as research lead for the duration of the project and to release staff time for data collection, including supporting the use of the accelerometers and, where appropriate, intervention implementation. Signed agreement is required from the care home manager and care home owner or representative.

A care home will be excluded if:

1) In the view of the research team it is not suitable for inclusion due to being subject to Care Quality Commission (CQC) enforcement notices, admission bans, or relevant moderate or major CQC compliance breaches;

2) It is receiving other special support for specific quality concerns, such as being currently subject to, or have pending, any serious safeguarding investigations, or receiving voluntary or compulsory admissions bans, or is in receipt of local commissioning special support due to quality concerns;

3) It has taken part in any of the earlier studies within the REACH programme;

4) It is taking part, has recently taken part, or is planning to take part, in another trial or initiative that conflicts with the REACH intervention or with the data collection during the course of trial involvement.
All residents in a care home will be exposed to the intervention should the home be allocated to the REACH intervention. Those considered eligible for individual outcome assessment will be permanent residents aged 65 years or over, not terminally ill nor permanently bedbound, and appropriately consented. A permanent resident is defined as residing in the care home and not in receipt of respite, day-care, or short-term rehabilitation.

\section{Care home recruitment and consent}

All residential care homes in a pre-specified catchment area of West Yorkshire will be identified via the CQC website and assessed for eligibility, as far as is possible, via publicly available information. Those deemed potentially eligible at this stage will receive an information sheet via the post with a reply slip to register interest in participation (a copy of this can be found in Additional file 2). Researchers will contact care homes to answer any queries and determine their interest if a reply slip is not returned within 2 weeks. For interested care homes, a researcher from the Academic Unit of Elderly Care and Rehabilitation (AUECR) will complete an initial eligibility assessment via telephone ahead of visiting the care home to determine full eligibility, obtain agreement to participate, and complete the recruitment process. Documented reasons for ineligibility and declining participation will be closely monitored by the trial team at each stage. The target is for a minimum of two care homes to be recruited per month.

To ensure the sample of 12 care homes is reached, in close liaison with Clinical Research Network staff, the Enabling Research in Care Homes (ENRICH; http:// enrich.nihr.ac.uk/) network will be contacted to assist with identifying homes in an additional catchment area. All care homes registered on the ENRICH network in the York and Harrogate areas of North Yorkshire will be sent the information sheet via email with contact details to register interest in participation. Colleagues from the local Clinical Research Network will follow-up contact with care homes if no response has been received within 2 weeks. For interested care homes, eligibility will be assessed by the study researcher via telephone and visit as outlined above.

\section{Resident recruitment and consent}

Following care home agreement to participate, all residents will be screened for eligibility. Demographic data will be recorded anonymously by the researcher through discussions with the care home manager and staff members who know the residents well. Residents will not be identified to the researcher by name, and any information provided will be differentiated only by a screening number.

An initial assessment of the capacity of each eligible resident to consent will be undertaken by the manager 
or nominated deputy. All residents will be assumed to have capacity unless assessed to lack capacity in accordance with the Mental Capacity Act (MCA) 2005 guidance [17]. Those with capacity will be provided with information about the study by the researcher and offered at least $24 \mathrm{~h}$ to consider participation if they wish. Formal written consent to take part will be sought by the researcher, and ongoing informal capacity assessments conducted by the researcher at each follow-up visit. If a resident's capacity is uncertain after initial assessment, the researcher will be asked to assist in determining capacity alongside a member of staff if the resident is willing (Fig. 2).

\section{Recruitment of those without capacity}

It is anticipated that many of the residents will lack the capacity to consent. The MCA requires that those lacking capacity are only included in research that is likely to be of direct benefit to those taking part or to benefit the particular population under study. In this trial, the intervention is delivered at the care home level, hence all residents may benefit directly from enhanced routine PA and indirectly through increasing social engagement. Excluding those without capacity would compromise the generalisability of findings by recruitment of an unrepresentative study sample and excluding this vulnerable population from the benefits of research evidence in improving practice.

Research Ethics Committee (REC) approval includes agreement to involve residents lacking capacity and the process to include them, namely to seek personal consultee or nominated consultee agreement for such residents to participate. For those residents who lose capacity during study participation following the provision of informed consent, an identified consultee will provide advice on a resident's continued involvement in the trial. Reasons for non-consent, ineligibility, or declining participation either by the resident themselves or their consultee will be closely monitored by the trial management team as part of a regular review of the recruitment process.

All resident and consultee information and consent documentation can be found in Additional file 2.

\section{Care home randomisation}

Care homes will be randomised on a 1:1 basis to the REACH intervention plus usual care or to usual care alone. Due to the small numbers of care homes (trial clusters), stratified randomisation will be implemented to ensure balance between the arms with respect to size of the care home (small/medium $\leq 40$ beds, large $>40$ beds) and whether or not an activity co-ordinator is in post (characteristics expected to be correlated with intervention implementation and outcome evaluation).
The allocation will be performed centrally by the statistician at the Clinical Trials Research Unit (CTRU) at the University of Leeds using a computer-generated minimisation program with a random element, following recruitment of residents and completion of their baseline assessments. CTRU will provide the random allocation to researchers involved in intervention implementation and adherence monitoring, so that all care home managers can be informed of their care home's allocation, and arrangements can be put in place for those homes randomised to intervention delivery. Researchers involved in the collection of outcome measures will not be informed of allocation, and efforts will be made to ensure that they remain blind. This will include maintaining separate office locations for 'blinded' and 'unblinded' researchers and requesting that care homes do not disclose their allocation to these researchers.

Due to the concern that smaller homes may not have enough residents providing baseline data to ensure sufficient outcome data for evaluation (despite initial assessment that there should be $8-12$ eligible residents at the care home), homes will only be randomised if there are at least five participating residents providing baseline data.

\section{Intervention and implementation process}

The REACH intervention is designed to be implemented and embedded in routine care delivery within the care home. It has been cumulatively built up from our earlier work and aims to achieve change in how all members of staff work with residents to encourage and support them to engage in more PA and spend less time sedentary. This will utilise the terminology 'moving more'. The intervention will focus on change in four domains of daily routines that embrace: independent/supervised movement of residents to get about; incorporating movement in social and leisure activities; providing opportunities for residents to engage in 'meaningful' activities; and encouraging residents to do as much of their own self-care and instrumental activities of daily living as is possible for them.

Implementation will comprise a cyclical service improvement approach. It will be led by a team involving key stakeholders in the change: staff, residents (if possible), and their relatives/friends facilitated by a senior member of care home staff. This synthesises a topdown and bottom-up approach to leading change [18]; this means that the active involvement of senior staff with the authority and legitimacy to drive the change process forward is aimed at securing organisational commitment to introducing and embedding the programme (topdown), and the engagement of those directly involved in action to deliver it ensures that their views and experiences will inform the pace and direction of change (bottom-up) [19, 20]. 


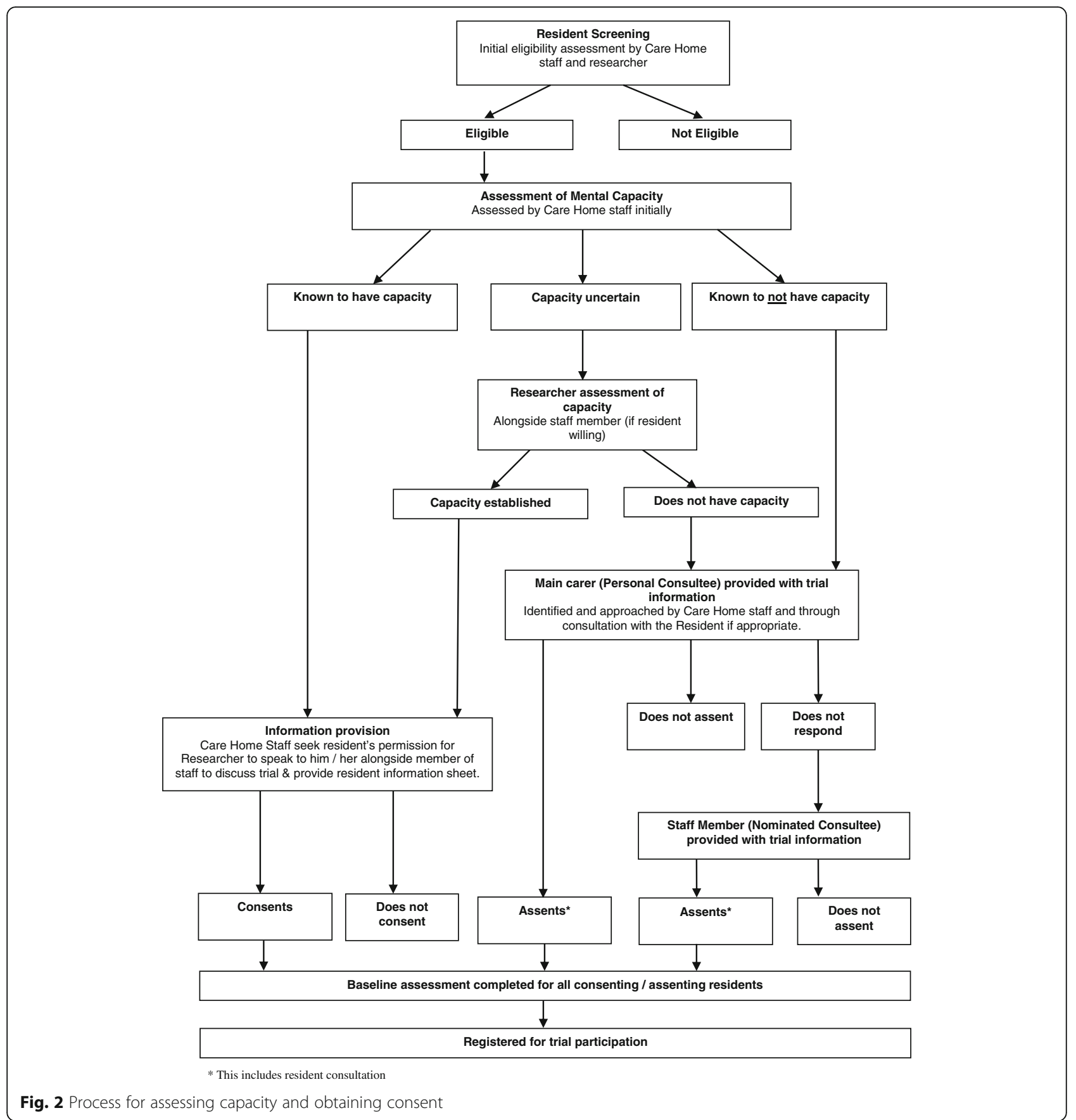

The intervention and implementation process does not prescribe particular kinds of changes that staff should make to increase residents' movement, but remains flexible to be adapted to each care home's needs.

Several strategies are in place to support implementation: identification of an intervention lead and core team in each home; provision of a manual, including an 'Ideas Bank' of resources to assist staff in getting started and keeping going; and training and support comprising a series of three interactive workshops provided individually to each home.

We have been guided by the template for intervention description and replication (TIDieR checklist [21]) and the treatment fidelity framework provided by the National Institutes of Health's Behavioral Change Consortium [22] to capture the intervention (its rationale, materials, procedures, etc.) and to guide approaches to implementation and fidelity monitoring. 


\section{Usual care}

Usual care is defined as 'normal' care delivered within the care home and will continue in both arms. No restrictions will be imposed on current practices or on care homes undertaking additional training and development as part of usual care. Details of general day-to-day activities will be recorded via researcher observations within public areas of a care home and completion of a pro-forma developed for the purposes of this study.

\section{Trial data collection}

The intention is that the intervention becomes embedded within the care home (if so randomised); thus it is important to assess the impact of the intervention on the care home as a whole. Consequently, data will be collected at the level of the care home (including staff) as well as from individual consenting residents at baseline (prior to randomisation) and at 3, 6, and 9 months post-randomisation. Data will be collected by trial researchers who will visit each participating care home at each follow-up time point. Personal information will be held centrally, in accordance with consent, by the research team to facilitate follow-up contact, but will be stored and processed separately to all other data collected for the purposes of the trial. In order to assess level of blinding, all outcome assessors will be asked to report immediately if they are unblinded.

A summary of all assessments to be used is provided in Table 1.

\section{Care home-level data}

The care home manager/nominated staff member will be asked to complete a care home booklet to provide information on the staff and resident profile of the home, and anonymous home-level data relating to hospital admissions, general practitioner (GP) call-outs, mortality rates, and falls in the last 3 months. At baseline, as part of the screening process, anonymous Physical Activity and Mobility in Residential Care Scale (PAM-RC) (Whitney et al. manuscript submitted) and Barthel Index [23] data will also be collected for all residents to establish the profile of PA, participation in activities of daily living/self-care at the level of the care home, and ambulatory capacity.

\section{Staff data}

At each data collection time point all staff who have faceto-face contact with residents, with the exception of those acting as a nominated consultee, will be asked to provide basic demographic data about themselves and complete a questionnaire regarding their experience of personcentred care provided in the care home (Person-centred Care Assessment Tool (P-CAT)) [24].

\section{Resident-level data}

Participating residents will be asked to complete questionnaires (via researcher interview) assessing their level of cognitive impairment (six-item Cognitive Impairment Test (6-CIT)) [25], mood (Geriatric Depression Scale (GDS)) [26], perceived health (EuroQol five dimensions (EQ-5D-5 L)) [27], and quality of life (dementia quality of life tool (DEMQoL)) [28]. Proxy completion of the EQ-5D questionnaire by a member of staff who knows the resident well will also be undertaken for all participating residents. Additionally, where residents are unable to answer the questions themselves, proxy completion of the DEMQoL questionnaire will be undertaken. Appropriate staff members will also be asked to complete questionnaires about physical function and mobility (Functional Ambulation Classification (FAC) and Elderly Mobility Scale (EMS])) of resident(s) they know well. Where possible, the same staff member will provide data for a particular resident throughout the study. Any staff member acting as a nominated consultee will not act as a staff proxy informant so that they remain independent of the research.

Residents who give their consent will be asked to wear an ActiGraph wGT3X-BT accelerometer (Actigraph, Pensacola, FL, USA) on the right hip, secured using an elasticated belt, during waking hours over the course of 7 days to record PA through movement counts collected over 60 -s epochs. Valid wear time will be defined as $\geq 8 \mathrm{~h} 25 \mathrm{~min}$ on $\geq 4$ days. For residents who provide valid data, the amount of time spent in various PA categories [29] and in sedentary behaviour will be identified using cut-points applied to the vertical axis accelerometer counts per minute (cpm) (see Table 2). A detailed protocol will be followed for administration of the accelerometers which will take place after the data collection defined above to ensure that accelerometer wear does not interfere with residents' routine PA levels and influence their questionnaire outcome assessments.

Adverse event data will be collected by a researcher on a monthly basis and the best method of collecting service use data will be explored, including data routinely recorded by the care home as well as, where possible, via receipt of hospital attendance data from NHS Digital and relevant Acute Hospital Trusts.

\section{Process evaluation}

A mixed-method approach will be employed with different stakeholders, at different levels (all homes; all intervention homes; purposive sub-sample of intervention and control homes).

Data will be collected across all 12 trial homes to describe usual care and the contextual factors within and outwith the care home environment that affect resident PA and sedentary time over the period of the trial. 
Table 1 Summary and timing of assessments

\begin{tabular}{|c|c|c|c|c|c|c|}
\hline \multirow[t]{2}{*}{ Assessment } & \multirow[t]{2}{*}{ Method of completion } & \multicolumn{5}{|l|}{ Timeline } \\
\hline & & Screening & Baseline & 3 months & 6 months & 9 months \\
\hline Care home eligibility & Researcher assessment & $x$ & & & & \\
\hline Resident screening (demographics) & Researcher assessment & $x$ & & & & \\
\hline $\begin{array}{l}\text { Physical Activity and Mobility in Residential } \\
\text { Care Scale (PAM-RC) and Barthel index }\end{array}$ & $\begin{array}{l}\text { Researcher interview/ } \\
\text { self-completion (S) }\end{array}$ & $x$ & & $x$ & $x$ & $x$ \\
\hline Resident consent (including consultee) & $\begin{array}{l}\text { Self-completion (R) } \\
\text { (witnessed) }\end{array}$ & $x$ & & & & \\
\hline Resident eligibility & Researcher assessment & $x$ & & & & \\
\hline $\begin{array}{l}\text { Contact details (including consultee, staff informant/ } \\
\text { proxy if applicable) }\end{array}$ & Researcher assessment & $x$ & & & & \\
\hline Care home demographics & Researcher interview (S) & & $x$ & $x$ & $x$ & $x$ \\
\hline Staff profile & Researcher interview (S) & & $x$ & $x$ & $x$ & $x$ \\
\hline $\begin{array}{l}\text { Home level mortality rates, hospital admissions, HCP contacts, and } \\
\text { adverse events }\end{array}$ & Researcher interview (S) & & $x$ & $x$ & $x$ & $x$ \\
\hline $\begin{array}{l}\text { Staff details questionnaire (including the } \\
\text { Person-centred Care Assessment Tool (P-CAT)) }\end{array}$ & Self-completed (S) & & $x$ & $x$ & $x$ & $x$ \\
\hline $\begin{array}{l}\text { Functional Ambulation Classification (FAC), and the } \\
\text { Elderly Mobility Scale (EMS) }\end{array}$ & $\begin{array}{l}\text { Researcher interview/ } \\
\text { self-completion (S) }\end{array}$ & & $x$ & $x$ & $x$ & $x$ \\
\hline Level of cognitive impairment (6-CIT) & Researcher interview (R) & & $x$ & $x$ & $x$ & $x$ \\
\hline Mood (Geriatric Depression Scale (GDS)) & Researcher interview (R) & & $x$ & $x$ & $x$ & $x$ \\
\hline Perceived health (EQ-5D-5 L) & $\begin{array}{l}\text { Researcher interview/ } \\
\text { proxy completion (R/SP) }\end{array}$ & & $x$ & $x$ & $x$ & $x$ \\
\hline Quality of life (DEMQOL) & $\begin{array}{l}\text { Researcher interview/ } \\
\text { proxy completion (R/SP) }\end{array}$ & & $x$ & $x$ & $x$ & $x$ \\
\hline Accelerometer measurements & Researcher assessment & & $x$ & $x$ & $x$ & $x$ \\
\hline Health Economics Questionnaire & $\begin{array}{l}\text { Researcher interview }(\mathrm{S}) / \\
\text { review of care notes }\end{array}$ & & $x$ & $x$ & $x$ & $x$ \\
\hline Service usage & Routine data sources & & Collectec & d throughou & & \\
\hline Hospital admissions/safety reporting & $\begin{array}{l}\text { Researcher assessment/ } \\
\text { routine data sources }\end{array}$ & & Collectec & d throughou & & \\
\hline Usual care review & $\begin{array}{l}\text { Researcher assessment } \\
\text { (observations) }\end{array}$ & & $x$ & $x$ & $x$ & $x$ \\
\hline Intervention delivery and adherence & Researcher assessment & & $x$ & $x$ & $x$ & $x$ \\
\hline
\end{tabular}

6-CIT Six-item Cognitive Impairment Test, DEMQOL Dementia quality of life tool, EQ-5D EuroQoL five dimensions, $H C P$, Health Care Professional $R$ resident, $S$ care home staff, SP staff proxy

Researchers will use a qualitative, trial-specific observational tool at each data collection time point to obtain insights into residents' PA at different times, in different locations, and in respect of residents with different types of need. They will also complete a chronology of

Table 2 Accelerometer cut-points

\begin{tabular}{lll}
\hline $\begin{array}{l}\text { Accelerometer counts } \\
\text { per minute }(\mathrm{cpm})\end{array}$ & PA classification & Examples of activities \\
\hline$<100 \mathrm{cpm}$ & Sedentary & Sitting, reclining \\
$100-759 \mathrm{cpm}$ & Low intensity PA & Upper body movements \\
$760-2019 \mathrm{cpm}$ & Light intensity PA & Self care, slow walk \\
$\geq 2020 \mathrm{cpm}$ & $\begin{array}{l}\text { Moderate-vigorous } \\
\text { (MV) PA }\end{array}$ & $\begin{array}{l}\text { Walking, sit-stand } \\
\text { transfers }\end{array}$ \\
\hline
\end{tabular}

PA physical activity organisational (including staffing) policy and practice changes that have occurred in the 3 months preceding data collection. Qualitative interviews will be conducted with a senior member of staff in each home at the conclusion of the trial by the dedicated process evaluation researcher(s) to explore the value attached to 'moving more' and how this may have changed over the period of the research.

Across all intervention homes, data will be collected on the intervention and implementation process by the process evaluation researcher and programme facilitators. This will include audio recordings of the workshops and collection of documentary data relating to the cyclical process of change over time (observation, action planning, and review sheets). Information will be 
gathered on which residents have been chosen for action and their characteristics; which domains of "moving more" have been targeted and with what effect; and which staff, occupying which roles, are nominated to provide support and encouragement to residents to engage in more PA. Qualitative interviews will be undertaken with the care home's intervention lead to explore how the intervention is understood, engaged with, and enacted by different stakeholders in the real life context of individual care homes, and examine how both intervention and contextual factors facilitate, delay, or impede change.

Within a sub-sample of two intervention and two control homes, ethnographic observations of levels of movement within public areas will be conducted by the process evaluation researcher(s) alongside informal discussion with residents about their experience of, and attitudes to, 'moving more', and interviews with staff generally about the value they attach to PA and practices in relation to it. These data will allow in-depth evaluation of how the intervention enhances attitudes towards and practices relating to PA among staff and residents over the study duration, over and above any changes that may be introduced within the control homes. Within the intervention homes, it will also contribute to understanding whether change occurs in the pattern of PA and sedentary behaviour over time, as well as in the engagement and reach of the intervention to staff and residents.

Within the sub-sample of two intervention homes, data on the theories of change and how these are enacted and impact on the pattern of movement over time will be collected through observation of implementation team meetings, review of documents relating to the service improvement cycle, and interviews with members of care staff, including the intervention lead, over the course of implementation. Since implementation is conceived of as a process and not a one-off event [30], documenting the process as it evolves over time and in relation to contextual factors in the care home is considered necessary. Analysis will be based on the principles of grounded theory [31].

\section{Economic evaluation}

A within-trial cost effectiveness analysis will be undertaken from the perspective of the NHS and Personal Social Care Sectors. It will compare costs and outcomes over the 9-month follow-up period between each arm. The analysis will use quality-adjusted life years (QALYs) derived from responses to the EQ-5D-5 L and EQ-5D-5 L proxy [32]. Cost estimates will be based on NHS resource use (accident and emergency/unplanned hospital admissions, falls, soft tissue injuries, NHS and non-NHS service use) collected from care notes and via a health economics questionnaire completed by researcher interviews with staff at 3, 6, and 9 months post-randomisation. The resources associated with implementation and delivery of the intervention will be included in the analysis. These will be based on routine data such as administrative records, trial-specific data collected during implementation, and a detailed description of the implementation and development process provided by the researchers involved in intervention implementation. Unit costs for health service resources will be obtained from national sources such as the Personal Social Services Research Unit (PSSRU), the British National Formulary (BNF), and NHS Reference cost database [33]. The non-parametric bootstrap method will be used to produce a within-trial probabilistic sensitivity analysis of the incremental cost effectiveness ratio. Longer term cost effectiveness will be estimated using a decision analytical model. The long-term cost effectiveness modelling will adopt the same perspective as the withintrial analysis and will follow good practice guidelines [34]. A value of information analysis will be undertaken [35].

\section{Sample size}

We aim to recruit 12 residential care homes (six per arm) with an average of 8-12 participating residents per home, based on recruitment in our earlier studies. A formal power calculation is not appropriate in this feasibility trial as effectiveness is not being evaluated. However, this target sample size allows us to maintain sufficient power for an effect size of 0.50 and above across the outcome measures while increasing the Type I error rate from 0.05 to 0.20 (Table 3). Increasing the Type I error rate provides assurance that the intervention is promising and warrants further evaluation given that we are making a preliminary and non-definitive randomised comparison of the intervention with usual care. It is recognised that, with an increased false-positive rate in this feasibility trial, a definitive trial may not show an intervention effect at the care home level; however, sufficient changes at an individual level may be detected which would also be of interest. The results generated from this study will be used to inform the sample size for a definitive trial.

Table 3 Anticipated power for a range of effect sizes across outcome measures with six clusters per arm, not accounting for losses to follow-up

\begin{tabular}{llll}
\hline Effect size & ICC & Power (cluster size =8) & Power (cluster size =12) \\
\hline 0.40 & 0.05 & $65 \%$ & $74 \%$ \\
& 0.10 & $59 \%$ & $65 \%$ \\
0.50 & 0.05 & $79 \%$ & $87 \%$ \\
& 0.10 & $72 \%$ & $78 \%$ \\
0.60 & 0.05 & $89 \%$ & $94 \%$ \\
& 0.10 & $83 \%$ & $88 \%$ \\
\hline
\end{tabular}

ICC intra-class correlation coefficient (which represents the amount of correlation between observations (residents) within a cluster (care home)) 


\section{Analysis}

Statistical analysis of the quantitative elements of the trial is the responsibility of the CTRU statistician, and a detailed statistical analysis plan will be written and agreed before data are analysed. No formal interim analyses are planned and final analysis will take place when all available data have been received and the database has been cleaned and locked. Analyses and data summaries will be conducted on an intention-to-treat population, defined as according to randomisation and regardless of non-compliance with the protocol or withdrawal from the study. The focus of analyses will be descriptive statistics and confidence interval (CI) estimation rather than formal hypothesis testing, with the exception of a preliminary estimate of effectiveness.

\section{Recruitment uptake and follow-up}

To assess feasibility of recruitment, the number of homes and residents screened, eligible, and providing consent/assent will be summarised, alongside reasons for non-participation. Retention during follow-up, including the number, timing, and reasons of withdrawals, will be reported overall and by study arm to assess whether there are any systematic differences between the arms which could be attributed to the intervention. The number of residents moving in and out of the care home will also be recorded.

\section{Intervention delivery and compliance}

Actual intervention delivery will be assessed using trialspecific documentation (case report forms), as well as through detailed process evaluation work. Documentation will allow reporting of the number of care homes failing to progress through implementation milestones and reasons for failure to implement any aspects of the intervention. This includes: the intervention workshops (including a summary of workshop content and attendees); the presence and content of action plans; and the level of support provided by the research team members responsible for intervention training to enhance intervention implementation. Implementation processes will be assessed in more depth via qualitative analysis of the process evaluation data, including observations of implementation team meetings, interviews with staff, informant conversations, and detailed field notes.

\section{Characterising usual care}

Specific initiatives relating to enhancing any part of resident care and any materials displayed in the care homes relating to PA (or the context of 'moving more'), such as leaflets or posters, will be summarised at each time point and by arm. Care home and staff characteristics will be summarised to inform the context in which the intervention was delivered, as well as staff turn-over resulting in employment at other trial care homes to assess the degree of contamination.

\section{Assessment of outcome measures}

The number and proportion of questionnaires received at each time point will be summarised overall and by study arm. Point estimates for resident scores will be calculated for each outcome measure, together with CIs for the differences in scores between the study arms. The amount of missing data will also be presented.

To inform the feasibility of monitoring levels of PA and sedentary behaviour, the number and proportion of residents who wear an accelerometer and the daily wear time will be summarised by arm at each time point, alongside the reasons for residents not wearing the accelerometer.

Preliminary estimate of the effectiveness of the intervention Through the use of accelerometers we are able to derive several different PA and sedentary behaviour end-points. We will be able to assess the amount of time residents spend sedentary and in low, light, and moderate to vigorous intensity physical activity (MVPA). We can also assess the number of daily bouts (defined as consecutive minutes) of specified lengths spent in these categories. Examples of such end-points include:

- Percentage of time spent sedentary and in low-intensity categories

- Number of daily sedentary bouts of $>60 \mathrm{~min}$

- Number of daily sedentary bouts of $>30 \mathrm{~min}$

- Number of daily bouts of low intensity $>5 \mathrm{~min}$

- Total length of time spent sedentary or in low-intensity physical activity

- The total time spent achieving any MVPA

For each PA outcome of interest (as detailed above) measured using the accelerometers, point estimates will be calculated by study arm based on cluster-level summaries, together with a range of CIs $(95 \%, 80 \%, 67 \%$, and $51 \%)$ to give a greater indication of the direction and effect size we might expect to see in a large-scale trial. Data will be reported separately at baseline and at each follow-up time point.

Following a review of the summaries for each of the PA outcomes, the most appropriate end-point for future use in the large-scale trial will be selected. This decision will be made on the basis of both the summary data and any emerging evidence on physiological effects (for example, that increasing the number of breaks in sedentary behaviour is more important than reducing the total amount of sedentary behaviour). No information will be provided split by treatment arm at this stage to avoid selection bias. Once this decision has been made we will then summarise these end-points by trial arm. 
A preliminary estimation of effectiveness will be carried out using methods appropriate for cluster randomised trials with a small number of clusters [36]. Point estimates of the 9-month outcome in each arm will be used to obtain difference or ratio estimates of the intervention effect depending on the type of outcome chosen. If the distribution of the summary measures in each arm is skewed, a logarithmic transformation will be considered. Cluster-level summaries will be used to obtain an $80 \% \mathrm{CI}$ for the intervention effect and hypothesis testing will be conducted at the $20 \%$ significance level using the $t$ test.

Adjustment for covariates will be carried out using a two-stage process. In the first stage, a standard regression model including the covariates of interest, but excluding the intervention effect, will be fitted to calculate cluster-specific expected values. Expected and observed values will be compared by computing a residual for each cluster. These cluster residuals will then be analysed using methods based on the $t$ test in the second stage of analysis.

To inform the sample size for a definitive trial, unadjusted and adjusted estimates of the ICC will be calculated alongside the amount of between-cluster variability in study outcomes and cluster size.

\section{Process evaluation}

Qualitative data (field notes, interviews, informant conversations, audio workshop recordings) will be analysed using grounded theory analytic strategies [31] combining simultaneous data collection and analysis, constant comparison, and search for negative cases. This approach provides a more robust, systematic, and in-depth approach to addressing issues of context and process over time, critical in this study.

Analysis will be conducted at several levels. For each care home, multiple sources of data will be drawn upon to provide a descriptive narrative account of the physical environment, organisation, staffing, resident profile, and pattern of PA and sedentary behaviour of residents. For intervention homes the process of understanding, engaging with, and implementing the intervention in the context of each care home will be analysed, drawing on documents and interviews with the intervention leads. Through comparison between intervention homes we will identify how the level of engagement with the intervention, the domains of action and the reach of the intervention across staff and residents vary, the factors that shape these, and how they affect the outcomes of the trial. Additional data relating to the actual process of implementation in time from the sub-sample of intervention homes will enable exploration of the theory of change underpinning the intervention and implementation process.

\section{Progression criteria for continuation to the definitive randomised controlled trial}

Guidelines for progression to a definitive RCT are based on a traffic light system of green (proceed to RCT design), amber (review RCT design and/or implementation, then proceed), red (stop and do not proceed), and are detailed in Additional file 3.

\section{Trial organisation and governance}

The REACH trial is sponsored by the Bradford Teaching Hospitals NHS Foundation Trust and is co-ordinated by the Academic Unit of Elderly Care and Rehabilitation (Bradford Teaching Hospitals NHS Foundation Trust and University of Leeds) and the CTRU (Clinical Trials Research Unit, University of Leeds). The trial management group consists of the co-applicants and the teams from the co-ordinating units. The study will be conducted in accordance with the Research Governance Framework for Health and Social Care (2005) and CTRU standard operating procedures.

Overall trial supervision is provided by the Programme Steering Committee (PSC), with an independent Chair and 'Patient and Public Involvement' (PPI) representation. A sub-group of the PSC will perform a safety monitoring function since a separate data monitoring and ethics committee is not required for a feasibility trial of this nature and duration.

Data will be entered, managed, and monitored for quality and completeness by the CTRU. Missing data (except individual items collected via questionnaires) will be chased until received, confirmed as not available, or the trial is at analysis. Data will be stored and managed in accordance with the provisions of the Data Protection Act (1998).

\section{Dissemination}

Results of the study will be published in peer-review publications and will be presented at national and international conferences. We will work with the PPI representatives to develop lay reports to disseminate research findings to resident and relative groups and the care home staff at participating homes.

Authorship will be agreed in accordance with the REACH Programme publication policy and in line with International Committee of Medical Journal Editors (ICMJE) recommendations.

\section{Discussion}

As the population ages the numbers at the oldest ages will increase the fastest. In 2008, there were 1.3 million people in the UK aged 85 years and over; this is projected to rise to 1.8 million by 2018 [37]. One consequence is an increase in demand for long-term care which, despite the increased emphasis on community 
care [38], will remain a necessary component of health and social care provision [2]. Residents of care homes are amongst the frailest in our population with significant health and social care needs [39, 40]. It is reported that older people [41] and care home residents undertake little PA $[4,42]$ and in fact spend the majority of their time sedentary [43]. Although "there is sufficient evidence to support a recommendation to reduce sedentary behaviour in older adults" [42], the majority of such work relates to younger people, and the implications for older adults are not yet robust. Decreasing levels of PA and increasing dependency have many adverse effects. Reduced PA and mobility problems compound health difficulties by directly affecting physical and psychological health, and reducing opportunities to participate in social activities [6].

We aim to address this challenge in our programme of work in which we are evaluating an intervention which actively engages staff within care homes to embed strategies to increase PA and decrease sedentary behaviour during the daily routines of the care home and its residents. This 'whole-home' intervention moves away from a model of time-limited provision of activities such as exercise classes.

Research in this setting in challenging; this feasibility trial will address important methodological issues, assess intervention implementation, and collect data to inform a definitive RCT to evaluate an intervention which addresses a central component of care for frail, elderly people. The work undertaken will also provide guidance and tested protocols for all research undertaken in the care home setting.

\section{Trial status}

The study commenced recruitment of care homes in August 2015, and recruitment of residents in December 2015; 138 residents at 11 care homes had been recruited as of 31 August 2016. The study is projected to complete recruitment by September 2016.

\section{Additional files}

Additional file 1: SPIRIT 2013 checklist: recommended items to address in a clinical trial protocol and related documents. (DOC $123 \mathrm{~kb}$ )

Additional file 2: REACH trial information and consent documentation. This document includes all information sheets and consent forms provided to the care home manager, residents, relatives, and staff members involved in the trial. (PDF $1306 \mathrm{~kb}$ )

Additional file 3: Progression criteria for continuation to the definitive randomised controlled trial. This document details guidelines for progression to a definitive randomised controlled trial $(\mathrm{RCT})$ which are based on a traffic light system of green (proceed to RCT design), amber (review RCT design and/or implementation, then proceed), or red (stop and do not proceed). (DOCX $20 \mathrm{~kb}$ )

\section{Abbreviations}

6-CIT: Six-item Cognitive Impairment Test; AUECR: Academic Unit of Elderly Care and Rehabilitation; BNF: British National Formulary; Cl: Confidence interval; CQC: Care Quality Commission; CTRU: Clinical Trials Research Unit;
DEMQoL: Dementia quality of life tool; EMS: Elderly Mobility Scale; ENRICH: Enabling Research in Care Homes; EQ-5D: EuroQoL five dimensions; FAC: Functional Ambulation Classification; GDS: Geriatric Depression Scale; GP: General practitioner; ICC: Intra-cluster correlation; MCA: Mental Capacity Act; METS: Metabolic equivalents; MVPA: Moderate-vigorous physical activity; NHS: National Health Service; NICE: National Institute for Health and Care Excellence; NIHR: National Institute for Health Research; PA: Physical activity; PAM-RC: Physical Activity and Mobility in Residential Care Scale; P-CAT: Personcentred Care Assessment Tool; PPI: Patient and Public Involvement; PSC: Programme Steering Committee; PSSRU: Personal Social Services Research Unit; QALY: Quality-adjusted life year; RCT: Randomised controlled trial; REACH: Research Exploring Physical Activity in Care Homes; REC: Research Ethics Committee

\section{Acknowledgements}

The authors wish to thank and acknowledge the ongoing support from the research team, care home staff and residents, and the Clinical Research Network who have contributed to setting up and running the REACH study. The authors would also like to thank the members of the Programme Steering Committee for their very helpful contribution throughout the REACH programme of research. We would like to acknowledge the ongoing expert support of the REACH Programme Grant Team which comprises: Professor Anne Forster, Head of the Academic Unit of Elderly Care and Rehabilitation, Bradford Teaching Hospitals NHS Foundation Trust and University of Leeds; Dr Karen Birch, Reader in Exercise Science, School of Biomedical Sciences, University of Leeds; Dr David R Ellard, Principal Research Fellow, Warwick Clinical Trials Unit, University of Warwick; Professor Amanda Farrin, Professor of Clinical Trials and Evaluation of Complex Interventions, University of Leeds; Mrs Joan Firth, PPI representative; Ms Beverley Gallagher, NHS Clinical Quality Manager for Care Homes and Domicilliary Providers; Ms Mary Godfrey, Reader in Health and Social Care, University of Leeds; Ms Liz Graham, Trial Manager; Dr John Green, Programme Manager, Academic Unit of Elderly Care and Rehabilitation; Dr Rebecca Hawkins, Lecturer in Qualitative Health Research, University of Leeds; Professor Claire Hulme, Professor of Health Economics, University of Leeds; Professor Rebecca Lawton, Professor in Psychology of Healthcare, University of Leeds; Dr Najma Siddiqi, Department of Health Sciences, Hull York Medical School, University of York \& Bradford District Care NHS Foundation Trust; Professor John Young, Academic Unit of Elderley Care and Rehabilitation, Bradford Teaching Hospitals NHS Foundation Trust and University of Leeds.

\section{Funding}

This research is funded by the National Institute for Health Research (NIHR) under its Programme Grants for Applied Research (PGFAR) Programme (Grant Reference Number RP-PG-1210-12017). The views expressed are those of the authors and not necessarily those of the NHS, the NIHR, or the Department of Health. There is funding body representation on the Programme Steering Committee (PSC), and progress reports have been submitted to NIHR on an annual basis since the grant commenced. NIHR oversees project execution in accordance with the grant proposal and timelines, and as such has not contributed to the running of the study nor the writing of this manuscript, save for review of the latter (for information only).

\section{Availability of data and materials}

Not applicable for this publication. As this is a feasibility trial to inform a definitive trial, sharing of the trial data set is not anticipated; however, any data requests should be sent to the corresponding author and would be subject to review by a sub-group of the trial team. All data sharing activities would require a data sharing agreement.

\section{Authors' contributions}

AFo is the lead grant holder, and oversees the design and implementation of the feasibility trial. JA takes a lead role in accelerometer data acquisition and analysis, participant recruitment, and data collection. KB takes a lead role in accelerometer data acquisition and analysis. $\mathrm{RC}$ is responsible for statistical analysis and the reporting of the trial. BC is responsible for statistical analysis and the reporting of the trial. AE is leading intervention training and implementation, and is responsible for care home recruitment. MG took a lead role in intervention development, leads intervention training and implementation, and is responsible for the implementation and analysis of the process evaluation. $L G$ is responsible for the operational delivery of the 
feasibility trial at CTRU. JG is responsible for the operational delivery of the REACH programme of research. $\mathrm{CH}$ leads the design of the health economic components of the trial and is responsible for the health economic analyses. $\mathrm{RL}$ took a lead role in intervention development. VM is responsible for the operational delivery of the trial at CTRU. NM was responsible for the operational delivery of the REACH programme of research and took a lead role in intervention development. AFa led the statistical and methodological design of the trial and is responsible for the main statistical analysis and reporting. All authors read and approved the final manuscript. This paper is written on behalf of the REACH Programme Team who meet regularly to oversee the programme of research. Team members have reviewed and agreed the content of the final manuscript.

\section{Authors' information}

AFo and MG: Academic Unit of Elderly Care and Rehabilitation, Bradford Institute for Health Research and University of Leeds. Bradford Institute for Health Research, Bradford Teaching Hospitals NHS Foundation Trust, Bradford, UK; JG, AE, and NM: Academic Unit of Elderly Care and Rehabilitation, Bradford Institute for Health Research, Bradford Teaching Hospitals NHS Foundation Trust, Bradford, UK; AFa, BC, RC, LG, and VM: Leeds Institute of Clinical Trials Research, University of Leeds, Leeds, UK; CH: Academic Unit of Health Economics, Leeds Institute of Health Sciences, University of Leeds, Leeds, UK; JA: School of Biomedical Sciences, University of Leeds, Leeds, UK. Academic Unit of Elderly Care and Rehabilitation, Bradford Institute for Health Research, Bradford Teaching Hospitals NHS Foundation Trust, Bradford, UK; KB: School of Biomedical Sciences, University of Leeds, Leeds, UK; RL: School of Psychology, University of Leeds, Leeds, UK. Yorkshire Quality and Safety, Bradford Institute of Health Research, Bradford Teaching Hospitals NHS Foundation Trust, Bradford, UK.

\section{Competing interests}

The authors declare that they have no competing interests.

\section{Consent for publication}

Not applicable.

\section{Ethics approval and consent to participate}

Ethical approval has been obtained through the East of England - Cambridgeshire and Hertfordshire Research Ethics Committee (REC) (Ref:15/EE/0125). Ethical approval includes agreed processes for obtaining consent (or assent) from (or on behalf of) all residents with and without capacity, in line with the provisions of the Mental Capacity Act. Any protocol amendments will be approved by the REC prior to informing researchers and care homes of any related process changes. Amendments impacting participants' involvement will be communicated to them in an appropriate manner, as agreed by the REC. At the time of writing, the current approved protocol is version 5 , dated 18 April 2016

\section{Publisher's Note}

Springer Nature remains neutral with regard to jurisdictional claims in published maps and institutional affiliations.

\footnotetext{
Author details

${ }^{1}$ Academic Unit of Elderly Care and Rehabilitation, Bradford Teaching Hospitals NHS Foundation Trust and University of Leeds, Bradford, UK. ${ }^{2}$ School of Biomedical Sciences, University of Leeds, Leeds, UK. ${ }^{3}$ Clinical Trials Research Unit, University of Leeds, Leeds, UK. ${ }^{4}$ Academic Unit of Elderly Care and Rehabilitation, Bradford Teaching Hospitals NHS Foundation Trust, Bradford, UK. ${ }^{5}$ Academic Unit of Health Economics, Leeds Institute of Health Sciences, University of Leeds, Leeds, UK. ${ }^{6}$ School of Psychology, University of Leeds, Leeds, UK.

Received: 17 October 2016 Accepted: 28 March 2017 Published online: 19 April 2017

\section{References}

1. Forder J, Fernandez J-L. Length of stay in care homes. Report commissioned by Bupa Care Services, PSSRU Discussion Paper2769. Canterbury: PSSRU; 2011

2. Harwood RH. Do we still need care homes? Age Ageing. 2004;33(6):529-30.
}

3. Office for National Statistics. Changes in the older resident care home population between 2001 and 2011. http://www.ons.gov.uk/ons/ dcp171776_373040.pdf. Accessed 20 Mar 2017.

4. Sackley CM, Levin S, Cardoso K, Hoppitt TJ. Observations of activity levels and social interaction in a residential care setting. Int J Ther Rehabil. 2006;13:370-3.

5. Caspersen CJ, Powell KE, Christenson GM. Physical activity, exercise, and physical fitness: definitions and distinctions for health-related research. Public Health Rep. 1985;100(2):126.

6. National Institute for Health and Care Excellence. Occupational therapy interventions and physical activity interventions to promote the mental wellbeing of older people in primary care and residential care. London: NICE; 2008.

7. Sedentary Behaviour Research Network. Letter to the editor: standardized use of the terms "sedentary" and "sedentary behaviours". Appl Physiol Nutr Metab. 2012;37(3):540-2.

8. Wilmot EG, Edwardson CL, Achana FA, Davies MJ, Gorely T, Gray LJ, et al. Sedentary time in adults and the association with diabetes, cardiovascular disease and death: systematic review and meta-analysis. Diabetologia. 2012;55(11):2895-905.

9. National Institute for Health and Care Excellence. Mental wellbeing of older people in care homes. UK: NICE quality standard [QS50]; 2013.

10. Crocker T, Forster A, Young J, Brown L, Ozer S, Smith J, et al. Physical rehabilitation for older people in long-term care. Cochrane Database Syst Rev. 2013;28(2):CD004294.

11. Kerse N, Peri K, Robinson E, Wilkinson T, von Randow M, Kiara L, et al. Does a functional activity programme improve function, quality of life, and falls for residents in long term care? Cluster randomised controlled trial. BMJ. 2008;337:a1445.

12. Katzmarzyk PT. Physical activity, sedentary behavior, and health: paradigm paralysis or paradigm shift? Diabetes. 2010;59(11):2717-25.

13. Bartholomew L, Parcel G, Kok G, Gottlieb N, Fernandez M. Planning health promotion program: an intervention mapping approach. 3rd ed. US: Wiley; 2011.

14. Moore GF, Audrey S, Barker M, Bond L, Bonell C, Hardeman W, Moore L, O'Cathain A, Tinati T, Wight D, Baird J. Process evaluation of complex interventions: Medical Research Council guidance. BMJ. 2015;350:h1258.

15. Century J, Cassata A, Rudnick M, Freeman C. Measuring enactment of innovations and the factors that affect implementation and sustainability: moving toward common language and shared conceptual understanding J Behav Health Serv Res. 2012;39(4):343-61.

16. Hawe P, Shiell A, Riley T. Theorising interventions as events in systems. Am J Community Psychol. 2009;43(3-4):267-76.

17. Johnston C, Liddle J. The Mental Capacity Act 2005: a new framework for healthcare decision making. J Med Ethics. 2007;33(2):94-7.

18. Sabatier PA. Top-down and bottom-up approaches to implementation research: a critical analysis and suggested synthesis. J Publ Policy. 1986:6(01):21-48.

19. Bate $P$, Mendel $P$, Robert G. Organizing for quality: the improvement journeys of leading hospitals in Europe and the United States. UK: Radcliffe Publishing; 2008.

20. Powell A, Rushmer $\mathrm{R}$, Davies $\mathrm{H}$. A systematic narrative review of quality improvement models in healthcare. UK: NHS Quality Improvement Scotland: 2009.

21. Hoffmann TC, Glasziou PP, Boutron I, Milne R, Perera R, Moher D, et al. Better reporting of interventions: template for intervention description and replication (TIDieR) checklist and guide. BMJ. 2014;348:g1687.

22. Borrelli B. The assessment, monitoring, and enhancement of treatment fidelity in public health clinical trials. J Public Health Dent. 2011;71(s1):S52-63.

23. Collin C, Wade D, Davies S, Horne V. The Barthel ADL Index: a reliability study. Int Disabil Stud. 1988;10(2):61-3.

24. Edvardsson D, Fetherstonhaugh D, Nay R, Gibson S. Development and initial testing of the Person-centered Care Assessment Tool (P-CAT). Int Psychogeriatr. 2010;22(01):101-8.

25. Brooke P, Bullock R. Validation of a 6 item cognitive impairment test with a view to primary care usage. Int J Geriatr Psychiatry. 1999;14(11):936-40.

26. Yesavage JA, Brink TL, Rose $\mathrm{TL}$, Lum $\mathrm{O}$, Huang $\mathrm{V}$, Adey $\mathrm{M}$, et al. Development and validation of a geriatric depression screening scale: a preliminary report. J Psychiatr Res. 1983;17(1):37-49.

27. Herdman M, Gudex C, Lloyd A, Janssen M, Kind P, Parkin D, et al. Development and preliminary testing of the new five-level version of EQ-5D (EQ-5D-5 L). Qual Life Res. 2011;20(10):1727-36. 
28. Smith S, Lamping D, Banerjee S, Harwood R, Foley B, Smith P, et al. Development of a new measure of health-related quality of life for people with dementia: DEMQOL. Psychol Med. 2007;37(5):737-46.

29. Hansen BH, Kolle E, Dyrstad SM, Holme I, Anderssen SA. Accelerometerdetermined physical activity in adults and older people. Med Sci Sports Exerc. 2012;44(2):266-72.

30. Fixsen DL, Naoom SF, Blase KA, Friedman RM. Implementation research: a synthesis of the literature. Tampa: University of South Florida, Louis de la Parte Florida Mental Health Institute, The National Implementation Research Network; 2005.

31. Charmaz K. Constructing grounded theory. USA: Sage; 2014.

32. Devlin NJ, Krabbe PF. The development of new research methods for the valuation of EQ-5D-5 L. Eur J Health Econ. 2013;14(1):1.

33. Curtis L, Burns A. Unit costs of health and social care. Personal Socia Services Research Unit Personal Social Services Research Unit. Canterbury: University of Kent; 2015.

34. Roberts M, Russell LB, Paltiel AD, Chambers M, McEwan P, Krahn M. Conceptualizing a model a report of the ISPOR-SMDM modeling good research practices task force-2. Med Decis Making. 2012;32(5):678-89.

35. Briggs A, Sculpher M, Claxton K. Decision modelling for health economic evaluation. Oxford: Oxford University Press; 2006.

36. Hayes RJ, Moulton LH. Cluster randomised trials. Boca Raton: CRC Press; 2009.

37. Wright E. 2008-based national population projections for the United Kingdom and constituent countries. Population Trends. 2010 Feb 1;139(1): 91-114.

38. Great Britain Department of Health. Our health, our care, our say: a new direction for community services. UK: The Stationery Office; 2006.

39. Continuing Care Conference. Care at the crossroads: CCC care census points the way forward. Continuing Care Coalition. www.ccc-ltc.org.uk/pr/2006-07-03.pdf. Accessed 20 Mar 2017.

40. Bowman C, Whistler J, Ellerby M. A national census of care home residents. Age Ageing. 2004;33(6):561-6.

41. Davis MG, Fox KR. Physical activity patterns assessed by accelerometry in older people. Eur J Appl Physiol. 2007;100(5):581-9.

42. Department of Health. Start active, stay active: a report on physical activity from the four home countries' Chief Medical Officers. London: Department of Health; 2011.

43. Holthe T, Thorsen $K$, Josephsson S. Occupational patterns of people with dementia in residential care: an ethnographic study. Scand J Occup Ther. 2007;14(2):96-107.

\section{Submit your next manuscript to BioMed Central and we will help you at every step:}

- We accept pre-submission inquiries

- Our selector tool helps you to find the most relevant journal

- We provide round the clock customer support

- Convenient online submission

- Thorough peer review

- Inclusion in PubMed and all major indexing services

- Maximum visibility for your research

Submit your manuscript at www.biomedcentral.com/submit

C) Biomed Central 